5 had above the sorbitol level $(0.9 \mathrm{~g}$ and $2.1 \mathrm{gram} / \mathrm{kg}$ respectively) Three patients received above the propylene glycol limit $(52.6 \mathrm{mg}$, $30 \mathrm{mg}$ and $190 \mathrm{mg} / \mathrm{kg}$ ), and at least one patient was getting $14.4 \mathrm{mg} /$ $\mathrm{kg}$ of hydroxybenzoates.

Conclusion When the information regarding quantities of excipients in medicines are available and calculated PICU patients are receiving significant amounts of excipients, some above the recommended safe limits.

\section{ADVERSE DRUG REACTIONS ASSOCIATED WITH CIPROFLOXACIN IN NEONATES}

doi:10.1136/archdischild-2012-302724.1639

${ }^{1} K A$ Kuruvilla, ${ }^{1} V$ James, ${ }^{2} \mathrm{M}$ Subramani, ${ }^{2 B}$ Mathew. 'Child Health \& Neonatology; ${ }^{2}$ Clinical Pharmacology, Christian Medical College \& Hospital Vellore, Vellore, India

Background Ciprofloxacin is used in many nurseries in developing countries. Data on drug concentrations and side effects of ciprofloxacin in neonates is limited.

Aims To study adverse drug reactions(ADR) associated with ciprofloxacin in term and preterm neonates and correlate them with drug levels.

Design Babies of 3 gestational age(GA) groups were enrolled: 37 (gp1), 32-36 (gp2) and 28-31 (gp3) weeks. Ciprofloxacin was administered twice daily at $10 \mathrm{mg} / \mathrm{kg} /$ dose IV. Lab parameters were done at baseline, day 3 and day 7, including peak and trough drug levels. Using Naranjo algorithm, babies who developed new symptoms after starting ciprofloxacin were classified into definite, probable, possible or doubtful ADR. Drug levels were correlated with ADR.

Results 165 babies receiving ciprofloxacin were enrolled. Predominant ADR were jaundice(79\%), rash(23\%), hyponatremia(28\%), anaemia(15\%) and hypokalemia(5\%). Using Naranjo algorithm, ProbableADR were cardiac arrhythmia, mucosal ulceration, renal failure and seizures. PossibleADR were rash, elevated liver enzymes, feed intolerance and leucopenia. ADRs were self-limited and treatable.

The mean (SD) trough and peak values of ciprofloxacin for the entire study population was $3.57(1.88)$ and 11.67 (3.66), respectively. Mean (SD) trough drug levels were gp 1-2.84 (1.54), gp 2-3.80 (1.9), gp 3-4.06 (1.98). GA did not seem to play a significant role in pharmacokinetics.

Drug levels higher than mean were seen in babies with rash, leucopenia and elevated LFT. Levels were adequate in babies with HIE, high in babies with NEC and lower in those with RDS.

Conclusions Ciprofloxacin can be considered safe for treating neonates.

\section{EFFICACY AND SAFETY OF MELATONIN IN NEONATES}

doi:10.1136/archdischild-2012-302724.1640

'S Aversa, 'L Marseglia, 'A Arco, 'G D'Angelo, 'E Cusumano, 'I Barberi, 'RJ Reiter, ${ }^{1} \mathrm{E}$ Gitto. 'Neonatal Intensive Care Unit, Department of Paediatrics, University of Messina, Messina, Italy; '2Department of Cellular and Structural Biology, The University of Texas Health Science Center, San Antonio, TX, USA

Background and Aims Newborns are more sensitive to oxidative stress than older infants. Melatonin, based on its properties as chronobiotic, antioxidant, or analgesic, offers perspectives of beneficial effects in neonatology. Aim of this study was to retrospectively review the efficacy and safety of melatonin administered at preterm and at term newborns in NICU.

Methods A retrospective patient record review of newborns treated with Melatonin in NICU of University of Messina (Italy) was performed.

Results 85 neonates were recruited and treated with Melatonin $(5-70 \mathrm{mg} / \mathrm{Kg} / \mathrm{die})$ in six previously published RCT. Melatonin has been given to 55 preterm infants with RDS and 30 at term newborns (10 with sepsis, 10 with perinatal asphyxia, and 10 with surgical abdominal malformations). That has always been given intravenously except for 10 septic newborns receiving oral administration. In our studies, melatonin treatment was able to reduce the level of proinflammatory cytokines, lipid peroxidation products and clinical parameters of inflammation and sepsis, and to improve the clinical outcome in terms of reduction of bronchodysplasia in preterm infants with RDS. None adverse event has been observed in our population of newborns treated with melatonin.

Conclusions To our knowledge, studies related to the toxicity of melatonin have not uncovered evidence of toxicity in humans even when given in very high doses. Our studies confirmed the potential role of melatonin as a treatment in different neonatal pathologies and the safety of its use in neonates at relatively high doses for short term and in various formulations.

\section{DIAZOXIDE OPENS THE CLOSING NEONATAL DUCTUS ARTERIOSUS}

doi:10.1136/archdischild-2012-302724.1641

K Momma. Pediatric Cardiology, Tokyo Women's Medical University, Tokyo, Japan

Background and Aims Sulfonylureas inhibit the ATP-sensitive potassium $\left(\mathrm{K}_{\mathrm{ATP}}\right)$ channel, are insulinogenic, and close the fetal ductus arteriosus. Diazoxide, a $\mathrm{K}_{\mathrm{ATP}}$ channel opener, is used for neonatal hyperinsulinemic hypoglycemia, and has been associated with the reopening of the ductus arteriosus. The aim of this study is to clarify ductus-opening effect of diazoxide.

Methods Neonatal rats were delivered by caesarian section nearterm and incubated at $34^{\circ} \mathrm{C}$. Diazoxide and pinacidil, another $\mathrm{K}_{\mathrm{ATP}}$ channel opener, were injected intraperitoneally immediately, or at one hour, or at four hours postnatally, and the ductus was studied 0.5 , and 1 hour later, with a rapid whole-body freezing method.

Results Diazoxide and pinacidil both induced hyperglycemia. Diazoxide and pinacidil delayed neonatal ductus closure following injection immediately after birth. At 2 hours, the control ductus was closed, whereas the ductus treated with $100 \mathrm{mg} / \mathrm{kg}$ of diazoxide at birth was widely patent with a diameter $40 \%$ of the fetal ductus. Ductus diameter at 60 minutes postnatally dilated from $10 \%$ to $40 \%$ with diazoxide. Diazoxide given to the closed ductus at 4 hours after birth did not open reopen it. The ductus was more sensitive to pinacidil than to diazoxide.

Conclusions Diazoxide and pinacidil open the closing ductus arteriosus of the neonatal rat. This study demonstrates that opening of $\mathrm{K}_{\mathrm{ATP}}$ channels results in opening of the ductus arteriosus, indicating that the $\mathrm{K}_{\text {ATP }}$ channel is physiologically and pharmacologically important in ductus opening. The ductus should be checked in the neonate before and after treatment with diazoxide.

\section{DESCRIBING THE USE OF OFF-LABEL AND NOT APPROVED MEDICATIONS IN A NEONATAL INTENSIVE CARE UNIT IN SOUTH BRAZIL}

doi:10.1136/archdischild-2012-302724.1642

${ }^{1} \mathrm{CG}$ Carvalho, ${ }^{2} \mathrm{M}$ Ribeiro, ${ }^{2} \mathrm{M}$ Bonilha, ${ }^{2} \mathrm{M}$ Fernandes Jr, ${ }^{2} \mathrm{RS}$ Procianoy, ${ }^{1} \mathrm{RC}$ Silveira. ${ }^{1}$ HCPA - UFRGS; ${ }^{2}$ UFRGS, Porto Alegre, Brazil

Background and Aims It is known that unlicensed medicines (unapproved) or used other way than directed in the label (off-label use) are widely prescribed in children. In the NICU, the severity of the patient justifies this type of prescription, evoking the risk-benefit ratio. We aimed to analyze the exposure to unapproved or off-label drugs in NICU in a tertiary university hospital in southern Brazil.

Method A descriptive cohort of drugs prescribed during hospitalization for 129 patients within 6 weeks. The drugs were classified as non-approved, and off-label for the dose, frequency, presentation, age or indication, according to FDA-approved e-lary. 